\title{
Gestión de la calidad de atención en laboratorios clínicos de hospitales públicos en Maracaibo, Venezuela
}

Managing the quality of attention in public hospitals' clinical laboratories in Maracaibo, Venezuela

Tania Molero ${ }^{1}$, Amelia Panunzio², Solbellys Cruz ${ }^{1}$, Milagros Núñez $^{3}$, Mariana Zambrano ${ }^{1}$, Irene Parra ${ }^{2}$ y Jesús Sánchez ${ }^{4}$

\footnotetext{
1 Departamento de Química. Escuela de Bioanálisis. Facultad de Medicina. Universidad del Zulia. tmp1509@hotmail.com, tmolero@gmail.com

2 Departamento de Salud Pública y Social. Escuela de Bioanálisis. Facultad de Medicina. Universidad del Zulia

3 Departamento de Morfofisiopatología. Escuela de Bioanálisis. Facultad de Medicina. Universidad del Zulia

4 Ingeniero Agrónomo. Universidad Nacional Experimental Politécnica de Maracaibo. Venezuela

Recibido 25 Febrero 2010/Enviado para Modificación 26 Septiembre 2010/Aceptado 15 Octubre 2010
}

\section{RESUMEN}

Objetivo Evaluar la gestión de calidad de los laboratorios según la perspectiva de sus usuarios externos, determinándose las dimensiones de la calidad relacionadas con su satisfacción y con las características sociodemográficas.

Métodos El estudio es descriptivo, la muestra estuvo conformada por 1875 pacientes de consulta externa que asistieron a laboratorios de hospitales públicos tipo III y IV nivel de atención ubicados en el área metropolitana del Estado Zulia, entre Octubre a Diciembre de 2008. Se aplicó una encuesta previamente validada. Para el análisis, se empleó distribución de frecuencias, análisis multivariante y análisis de la varianza.

Resultados Los resultados mostraron que la mayoría de los usuarios son pacientes de sexo femenino (72,7\%), de procedencia local (87,9\%); en edades entre 15 y 45 años $(65,7 \%)$ y con grado de instrucción primaria y secundaria $(70,5 \%)$. El análisis multifactorial demostró que el componente Gestión de Calidad, puede ser valorado por las dimensiones: Accesibilidad, Elementos tangibles, Capacidad de Respuesta, Competencias profesionales y Seguridad. Hubo diferencias altamente significativas $(p<0,001)$ entre los distintos grados de instrucción y la edad al calificar la gestión de calidad. Se detectaron debilidades en cuanto a tiempo de espera en recepción, comodidad de la sala de espera y uso de medidas de seguridad del personal técnico.

Conclusión Los resultados permitirán establecer acciones tendentes a evaluar el grado de mejoramiento del servicio y las metas propuestas en pro de mejorar la calidad de atención, medidas que formarán parte de un programa de aseguramiento de la calidad conforme la normativa internacional.

Palabras Clave: Gestión de calidad, satisfacción del paciente, laboratorios (fuente: DeCS, BIREME) 


\section{ABSTRACT}

Objective Evaluating how clinical laboratories' quality was being managed according to the view of external users, thereby determining the dimensions of quality related to their satisfaction and sociodemographic characteristics.

Methods The study was descriptive; the sample consisted of 1,875 outpatients attending the laboratories of type 3 and 4 care level public hospitals located in the metropolitan area of Zulia State in Venezuela between October and December 2008. A previously validated survey was applied. Frequency allocation, multivariate analysis and variance analysis were used for analysing data.

Results The results showed that the most users were female $(72.7 \%)$, of local origin (87.9\%), aged between 15 and $45(65.7 \%)$ and had received primary and secondary education $(70.5 \%)$. Multivariate analysis showed that quality management could be evaluated in terms of accessibility, tangible elements, response ability, safety and professional competence. There were highly significant differences $(p<0.001)$ between educational level and age when evaluating management quality. Weaknesses were revealed concerning time spent waiting in reception, waiting room comfort and safety measures used by technical staff.

Conclusions The results should lead to establishing action aimed at evaluating the degree of improvement in service and proposed targets for improving the quality of attention; such measures will form part of a quality assurance programme in accordance with international standards.

Key Words: Quality management, patient satisfaction, laboratory (source: $\mathrm{MeSH}$, $N L M)$.

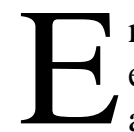

$\mathrm{n}$ la actualidad las organizaciones del sector público y privado se encuentran inmersas en un mundo completamente globalizado que exige altos niveles de competitividad. En este entorno, la calidad del producto o servicio constituye una estrategia de diferenciación y excelencia reflejada en mayor número de usuarios o clientes y mejor imagen de la empresa (1). Se garantiza aún más el éxito si toda institución adopta normas que le sirvan como marco de referencia; asociadas éstas a normas internacionales de calidad sustentadas y aceptadas por principios de probada eficacia (2).

En este contexto, toda empresa dedicada al servicio, debe asegurar mediante la mejora continua que ese servicio cubra las expectativas y necesidades del cliente o usuario (3). Para cubrir esta exigencia, debe incrementar la Gestión de Calidad desarrollando una serie de acciones planificadas y dirigidas a la dirección, políticas y estrategias, gestión de los recursos y de las personas, y debe orientar al usuario todos los procesos de la organización alcanzando un impacto positivo en la sociedad (4). 
A nivel del sector salud, la calidad y la eficiencia constituyen requisitos indispensables para mantener un ambiente competitivo desde el punto de vista social, laboral y tecnológico. Con la reforma de los procesos sanitarios que actualmente viven la mayoría de países en Latinoamérica, la percepción que tienen los usuarios sobre los servicios de salud se ha considerado como un aspecto importante para la formulación de políticas sociales y, en particular, de políticas públicas de salud, aceptando que un mayor grado de satisfacción, asociado a percepciones de mayor calidad, es un resultado social deseable por la gestión hospitalaria (5).

Dentro de las instituciones de salud, los laboratorios de atención pública, generan productos y servicios al paciente, a la comunidad, a su mismo personal, a las instituciones y autoridades de salud y a otras empresas, por lo que las exigencias de la salud y la seguridad, así como los requisitos legales y las leyes del mercado, obligan a los mismos a incorporar el concepto de calidad en sus rutinas diarias (6).

Dada la importancia fundamental que tiene la evaluación de la gestión de la calidad en los laboratorios clínicos, resulta indispensable la realización de estudios diagnósticos a través de instrumentos confiables que permitan establecer planes estratégicos en pro de una mejora de la calidad de las mismas. Es por ello que, el propósito de la presente investigación fue evaluar la Gestión de Calidad de Laboratorios Clínicos de Hospitales de Atención Pública ubicados en el área metropolitana del Estado Zulia, desde la perspectiva del usuario, determinándose las dimensiones de la calidad relacionadas con su satisfacción y con las características sociodemográficas, cuyos aportes permitan facilitar acciones para elevar los niveles de satisfacción institucional.

\section{MÉTODOS}

La investigación se enmarcó en un estudio de tipo descriptivo y diseño transversal.

Las dimensiones de la calidad propuestas en la presente investigación son aquellas relacionadas a la calidad de atención del paciente en recepción, toma de muestra y entrega de resultados: accesibilidad; elementos tangibles; capacidad de respuesta; competencia profesional y seguridad. Estas a su vez, resultaron de análisis propuestos por autores con amplia experiencia en valorar calidad del servicio; tales como Parasuraman, Zeithaml y Berry (7); Villegas y Rosa (8) y Varo (9). 
La población estuvo representada por pacientes mayores de 15 años, provenientes de consulta externa y que asisten a los laboratorios de planta de los hospitales públicos de III y IV nivel de atención del área metropolitana del Estado Zulia que cubren una área de influencia de 1500000 habitantes.

Se practicó un muestreo probabilístico aleatorio estratificado, habiéndose obtenido una muestra de 1875 pacientes. El error muestral fue de 3,6 \% con un nivel de confianza de $95 \%$.

Para la obtención de los datos se diseñó y empleó un instrumento que contenía 21 afirmaciones que eran contestadas según una escala de valoración con numerales del 1 al 5. La alternativa 5 denota estar totalmente de acuerdo con la afirmación y la 1 estar totalmente en desacuerdo. Fue sometido a prueba de validez de contenido por expertos en el área y se realizó una prueba piloto con el propósito de medir la confiabilidad, empleándose el coeficiente Alfa de Cronbach, resultando un coeficiente de 0,895 , lo que califica al instrumento como confiable para ser aplicado en la investigación.

Los pacientes fueron entrevistados durante los meses de Octubre a Diciembre del año 2008, aplicándose el instrumento bajo la modalidad de encuesta en el momento en que el paciente retiraba el informe de resultados, e incluyó 3 aspectos de la calidad de atención: recepción y sala de espera, toma de muestras y entrega de resultados.

Los datos se procesaron utilizando el programa estadístico SPSS v. 10 empleando para el análisis, pruebas de significancia estadística, t de Student y ANOVA para establecer la relación entre las características sociodemográficas de la muestra y las dimensiones de la calidad con la Gestión de Calidad. Con el fin de determinar si las dimensiones propuestas guardan relación con la gestión de calidad, se aplicó análisis multifactorial, con método de extracción de componentes principales y rotación Varimax con Kaiser. Para identificar las áreas críticas de la calidad de atención tendentes a ser mejoradas; se empleó la media como medida de tendencia central.

\section{RESULTADOS}

En relación a las características socio demográficas de los usuarios un $72,7 \%$ de los pacientes que acuden a estos laboratorios corresponden al sexo femenino; con respecto a la edad, el 65,7 \% de los usuarios posee una edad que oscila entre 15-25 años. En cuanto a la procedencia y al grado de 
instrucción, el 87,9 \% de los usuarios son de procedencia local y en relación al grado de instrucción, el 45,8\% de los pacientes refirieron poseer un nivel de estudios secundario mientras que un $24,7 \%$ instrucción primaria. (resultados no presentados).

En el presente estudio, con la finalidad identificar las dimensiones de la Gestión de Calidad relacionadas con la satisfacción del paciente como usuario externo de los servicios de laboratorio clínico, se efectuó el análisis factorial empleando la matriz de componentes principales y rotación varimax con Kaiser. Como resultado del mismo se obtuvieron 5 factores cuya composición se muestra en la Tabla 1. Los Componentes 1, 2 y 3 incluyen 4 variables de análisis cada uno; y los componentes 4 y 5 incluyen 2 variables.

Tabla 1. Composición de las dimensiones de Gestión de Calidad Matriz de Componentes Rotados (a)

\begin{tabular}{|c|c|c|c|c|c|}
\hline \multirow[b]{2}{*}{ Variables de Análisis } & \multicolumn{5}{|c|}{ Componentes } \\
\hline & 1 & 2 & 3 & 4 & 5 \\
\hline R5: Trato del personal de atención al paciente & 0.817 & & & & \\
\hline R6: Instrucciones previas a toma de muestra & 0.729 & & & & \\
\hline R7: Tiempo de espera en recepción & 0.617 & & & & \\
\hline R12: Trato del personal en recepción de muestras & 0.558 & & & & \\
\hline R10: Iluminación de recepción y toma de muestras & & 0.783 & & & \\
\hline R8: Higiene y limpieza de ambientes & & 0.708 & & & \\
\hline R9: Temperatura ambiental del laboratorio & & 0.677 & & & \\
\hline R11: Comodidad de la sala de espera & & 0.601 & & & \\
\hline R18: Horario asignado para la entrega de resultados & & & 0.839 & & \\
\hline R17: Tiempo de espera para la entrega de resultados & & & 0.828 & & \\
\hline R19: Trato del personal que entrega de resultados & & & 0.480 & & \\
\hline R20: Claridad en el reporte de resultados & & & 0.370 & & \\
\hline R13: Destreza del personal de toma de muestra & & & & 0.831 & \\
\hline R14: Comodidad del área de toma de muestras & & & & 0.494 & \\
\hline R15: Uso de guantes por el personal técnico & & & & & 0.833 \\
\hline R16: Uso de bata por el personal técnico & & & & & 0.756 \\
\hline
\end{tabular}

Dentro de este mismo orden de ideas, como resultado del Análisis Factorial por rotación, a continuación se demuestra en la Tabla 2, que los primeros 3 componentes tienen un auto-valor superior a $1(1,021 ; 1,018$ y 1,008 respectivamente); los cuales explican el $60,9 \%$ de la varianza. Se destaca que, los otros 2 factores restantes, tienen un auto-valor muy cercano a $1(0,985$ y 0,969$)$ respectivamente.

Relacionando los resultados de las Tablas 1 y 2, el componente $1(20,4 \%$ de la varianza total), podría explicarse como Accesibilidad, el cual define los ítems del instrumento con mayor peso, tales como los referidos al trato del 
personal encargado de recepción y toma de la muestra, tiempo de espera para ser atendido y la disposición de parte del personal de resolver dudas y sugerencias.

Tabla 2. Varianza Total Explicada por los componentes detectados por el Análisis Factorial para las dimensiones de la Gestión de Calidad

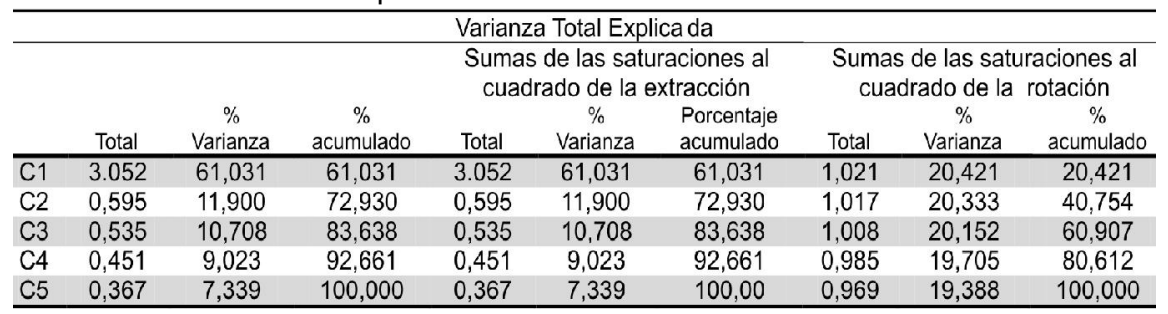

El factor o componente 2, que explica el 20,3\% de la varianza total, está relacionado con los Elementos tangibles, orientados éstos a la limpieza, iluminación y temperatura ambiental de las áreas, así como también a la comodidad de la sala de espera.

El factor 3 explica el 20,1\% de la varianza total y se ha denominado Capacidad de Respuesta, que incluye aspectos referentes a la entrega de resultados, tales como tiempo de espera, trato del personal, horario designado y claridad de resultados.

El componente 4 (19,7\% de la varianza total), corresponde a la Competencia profesional orientada a la calidad técnica del personal encargado de la toma y recepción de la muestra.

Tabla 3. Matriz de componentes para las dimensiones de la Gestión de Calidad

\begin{tabular}{lccccc}
\hline & Matriz de Componentes Rotados(a) & & \\
& 1 & 2 & 3 & 4 & 5 \\
\hline Accesibilidad & 0,929 & & & & \\
Elementos Tangibles & & 0,921 & & & \\
Capacidad de respuesta & & & 0,908 & 0,889 & \\
Competencias Profesionales & & & & 0,880 \\
\hline Seguridad
\end{tabular}

Método de extracción: Análisis de componentes principales. Método de rotación: Normalización Varimax con Kaiser. (a) La rotación ha convergido en 6 iteraciones

Finalmente, el componente 5 (19,3\% de la varianza total) está relacionado a la Seguridad, en términos del uso por parte del personal técnico de guantes y bata de laboratorio, factores éstos que hacen que el paciente perciba al momento de la toma de la muestra un proceso seguro y confiable. 
La Matriz de Componentes expresada en la Tabla 3, indica que el factor Gestión de Calidad, está configurado por las dimensiones: Seguridad, Capacidad de Respuesta, Accesibilidad, Competencias profesionales y elementos tangibles. Allí, queda de manifiesto que la Accesibilidad y los Elementos Tangibles tienen cargas factoriales altas (0,929 y 0,921 respectivamente). Las Dimensiones: Capacidad de Respuesta, Competencias Profesionales y Seguridad tienen cargas factoriales mayores a $0,81(0,908 ; 0,889$ y 0,880 respectivamente); siendo éstas satisfactorias.

$\mathrm{Al}$ relacionar las características sociodemográficas y las dimensiones de la calidad con la Gestión de Calidad en los laboratorios clínicos estudiados se pudo observar que los resultados del Análisis de la Varianza (ANOVA) demuestran que se detectan diferencias significativas en la Gestión de Calidad y Elementos Tangibles según la edad (F significativo a p $<0,05)$ como se muestra en la Tabla 4.

Se obtuvieron diferencias estadísticamente significativas entre las ponderaciones de la Gestión de Calidad según el Grado de Instrucción. Como producto de aplicar el ANOVA sobre la data se detectan diferencias altamente significativas entre los distintos grados de instrucción en razón de la media de ponderación de la calidad de gestión, dado que el valor de $\mathrm{F}=3,49$ es significativo a un valor menor a 0,01 . De igual forma se encontraron diferencias para las ponderaciones de los Elementos Tangibles $(\mathrm{F}=7,54$; sig. $<0,001)$ y las competencias Profesionales ( $\mathrm{F}=4,72$; sig. $<0,001$ ) los cuales pudieran ser las dimensiones cuya variación según la formación originen cambios en la percepción de la calidad.

En relación a la determinación de las áreas críticas o debilidades de la calidad de gestión, se procede a determinar cuales son las preguntas catalogadas como tales a partir de las medias y desviaciones típicas obtenidas. Las preguntas con menor calificación denotan insatisfacción por parte de los usuarios y corresponden a las relacionadas con el tiempo de espera del paciente para ser atendido en recepción, ambientación y comodidad la sala de espera y uso de guantes por parte del personal técnico al momento de la toma de la muestra (con una media de 3,94; 3,06 y 3,97 respectivamente). Estos aspectos forman parte de los atributos que describen a las dimensiones accesibilidad, elementos tangibles y seguridad. 
Tabla 4. ANOVA para las medias de Gestión de Calidad y sus dimensiones según la Edad y el Grado de Instrucción

\begin{tabular}{|c|c|c|c|c|c|c|}
\hline \multicolumn{7}{|c|}{ Edad } \\
\hline & Fuente & $\begin{array}{c}\text { Suma } \\
\text { de los } \\
\text { cuadrados }\end{array}$ & $\begin{array}{c}\text { Grados } \\
\text { de } \\
\text { libertad }\end{array}$ & $\begin{array}{c}\text { Media } \\
\text { Cuadrática }\end{array}$ & $\mathrm{F}$ & SIG. \\
\hline \multirow{3}{*}{ Accesibilidad } & Inter-grupos & 6,15 & 4 & 1,540 & 1,986 & 0,095 \\
\hline & Intra-grupos & 558,2 & 720 & 0,775 & & \\
\hline & Total & 564,4 & 724 & & & \\
\hline \multirow{3}{*}{$\begin{array}{l}\text { Elementos } \\
\text { Tangibles }\end{array}$} & Inter-grupos & 7,8 & 4 & 1,958 & 3,525 & 0,007 \\
\hline & Intra-grupos & 399,9 & 720 & 0,555 & & \\
\hline & Total & 407,7 & 724 & & & \\
\hline \multirow{3}{*}{$\begin{array}{c}\text { Competencia } \\
\text { Profesional }\end{array}$} & Inter-grupos & 1,5 & 4 & 0,398 & 1,190 & 0,314 \\
\hline & Intra-grupos & 241,0 & 720 & 0,335 & & \\
\hline & Total & 242,6 & 724 & & & \\
\hline \multirow{3}{*}{$\begin{array}{c}\text { Capacidad de } \\
\text { Respuesta }\end{array}$} & Inter-grupos & 5,3 & 4 & 1,346 & 2,050 & 0,086 \\
\hline & Intra-grupos & 472,6 & 720 & 0,657 & & \\
\hline & Total & 242,6 & 724 & & & \\
\hline \multirow{3}{*}{ Seguridad } & Inter-grupos & 2,2 & 4 & 0,551 & 1,317 & 0,262 \\
\hline & Intra-grupos & 301,3 & 720 & 0,418 & & \\
\hline & Total & 303,5 & 724 & & & \\
\hline \multirow{3}{*}{$\begin{array}{c}\text { Gestión de } \\
\text { Calidad }\end{array}$} & Inter-grupos & 9,6 & 4 & 2,417 & 2,436 & 0,046 \\
\hline & Intra-grupos & 714,3 & 720 & 0,992 & & \\
\hline & Total & 724,0 & 724 & & & \\
\hline \multicolumn{7}{|c|}{ Grado de Instrucción } \\
\hline \multirow{3}{*}{ Accesibilidad } & Inter-grupos & 5,9 & 4 & 1,482 & 1,910 & 0,107 \\
\hline & Intra-grupos & 558,4 & 720 & 0,776 & & \\
\hline & Total & 564,4 & 724 & & & \\
\hline \multirow{3}{*}{$\begin{array}{l}\text { Elementos } \\
\text { Tangibles }\end{array}$} & Inter-grupos & 16,4 & 4 & 4,103 & 7,549 & 0,001 \\
\hline & Intra-grupos & 391,3 & 720 & 0,544 & & \\
\hline & Total & 407,7 & 724 & & & \\
\hline \multirow{3}{*}{$\begin{array}{c}\text { Competencia } \\
\text { Profesional }\end{array}$} & Inter-grupos & 6,2 & 4 & 1,551 & 4,723 & 0,001 \\
\hline & Intra-grupos & 236,4 & 720 & 0,328 & & \\
\hline & Total & 242,6 & 724 & & & \\
\hline \multirow{3}{*}{$\begin{array}{c}\text { Capacidad de } \\
\text { Respuesta }\end{array}$} & Inter-grupos & 3,8 & 4 & 0,966 & 1,467 & 0,211 \\
\hline & Intra-grupos & 474,2 & 720 & 0,659 & & \\
\hline & Total & 478,0 & 724 & & & \\
\hline \multirow{3}{*}{ Seguridad } & Inter-grupos & 3,3 & 4 & 0,833 & 1,999 & 0,093 \\
\hline & Intra-grupos & 300,1 & 720 & 0,417 & & \\
\hline & Total & 303,5 & 724 & & & \\
\hline \multirow{3}{*}{$\begin{array}{c}\text { Gestión de } \\
\text { Calidad }\end{array}$} & Inter-grupos & 13,7 & 4 & 3,433 & 3,490 & 0,008 \\
\hline & Intra-grupos & 710,2 & 720 & 0,986 & & \\
\hline & Total & 724,0 & 724 & & & \\
\hline
\end{tabular}

DISCUSIÓN

Los resultados permitieron caracterizar a la población estudiada desde los aspectos sociodemográficos. La mayoría de los usuarios que asisten a estos centros asistenciales son de sexo femenino, con grado de instrucción primaria y secundaria, en edades comprendidas entre 15 y 45 años de edad y proceden de la misma ciudad donde se encuentran ubicados los centros hospitalarios. Estos resultados coinciden con los de otras investigaciones, donde un alto 
porcentaje de los pacientes pertenecen al género femenino, catalogados como adultos jóvenes, de procedencia local y con formación primaria y secundaria $(10,11)$.

Con relación a las dimensiones propuestas para medir la calidad de los servicios de laboratorio desde la perspectiva de los usuarios externos, el análisis multifactorial, demostró que el componente Gestión de Calidad, puede ser valorado a través de las dimensiones: Accesibilidad, Elementos tangibles, Capacidad de Respuesta, Competencias profesionales y Seguridad. El análisis estadístico mostró que las cargas factoriales fueron altas, superiores a 0,81 ; lo cual demuestra que las cinco dimensiones tienen relación con la Gestión de Calidad. Considerando la varianza total explicada para las dimensiones de la Gestión de Calidad propuestas, las tres primeras: Accesibilidad, Elementos tangibles y Capacidad de Respuesta explican el 60,9 \% de la varianza total, porcentaje que dentro de una investigación no experimental dentro del ámbito de las ciencias sociales es considerado como satisfactorio (12).

Relacionando las características sociodemográficas y las dimensiones de la calidad con la Gestión de Calidad, los resultados mostraron que en cuanto a la edad hubo diferenciación en la concepción de la variable estudiada, donde las personas mayores de 36 años asignan menor puntaje a la dimensión elementos tangibles en comparación con los grupos etarios de menor edad. En líneas generales se observa que la edad es inversamente proporcional a la valoración de la Calidad de Gestión, es decir que a medida que aumenta la edad del paciente, menor es la asignación que le da a las preguntas realizadas.

Se detectan diferencias altamente significativas entre los distintos grados de instrucción al valorar la calidad de atención. En este estudio, es clara la tendencia que a medida que se eleva la formación o nivel de instrucción, mayor es el nivel de exigencia por parte de los pacientes al solicitar el servicio, reflejándose principalmente en las dimensiones competencia profesional y elementos tangibles; resultados que coinciden con otros investigadores (13-15).

Los resultados mostraron que el tiempo de espera del paciente para ser atendido en recepción, condiciones ambientales, comodidad de la sala de espera y uso de guantes por parte del personal técnico al momento de la toma de la muestra constituyen las debilidades o áreas críticas tendentes a ser mejoradas. Estos hallazgos coinciden con los realizados por otros autores al reportar las debilidades frecuentes del servicio de atención médica y de laboratorio cuando valoran la calidad de atención según la percepción de sus usuarios (16-19). 
Los aportes de esta investigación proponen acciones que permitan replantear mecanismos de atención hacia el paciente y mejorar el ambiente de trabajo de todo el personal, determinar la medida o grado de mejoramiento de los servicios y evaluar el cumplimiento de las propuestas y metas en pro de mejorar la calidad de atención de nuestras instituciones de salud *

Agradecimientos: Al personal de los laboratorios públicos participantes. A los pacientes por su amplia colaboración. Fuentes de financiación: este proyecto fue financiado por la Universidad del Zulia.

\section{REFERENCIAS}

1. Llorens F, Fuentes M. Gestión de la Calidad Empresarial, Fundamentos e Implantación. Madrid. España. Ediciones Pirámide; 2005.

2. Franklin F. Enrique. Auditoría Administrativa. Primera Edición. México. Editorial McGraw Hill Interamericana. 2001. p 559.

3. Gutiérrez Pulido H. Calidad Total y Productividad. Segunda Edición. México: Editorial McGraw Hill Interamericana; 2005. p 409.

4. Zeithaml VA, Bitner M. Marketing de Servicios. Segunda Edición. México. Editorial McGraw Hill Interamericana; 2004.

5. Cobra M. Marketing de Servicios. Segunda Edición. Bogotá. Colombia. Editorial McGraw Hill Interamericana; 2001. p. 140-148.

6. Fernández C, Mazziotta D. Gestión de Calidad en el Laboratorio Clínico. Confederación Latinoamericana de Bioquímica Clínica (Colabiocli). Editorial Médica Panamericana. Buenos Aires, Argentina; 2005.

7. Zeithaml VA, Parasuraman A, Berry LL. SERVQUAL: a multiple-item scale for measuring consumer perceptions of service quality. Journal of Retailing. 1988; 64(1):12-40.

8. Villegas M, Rosa I. La Calidad Asistencial: Concepto y Medida. Revista de Dirección y Administracion de Empresas Cepade de la Universidad de Sevilla. 2003; 29: 50-58.

9. Varo J. Gestión Estratégica de la Calidad de los Senvicios Sanitarios. Un modelo de gestión hospitalaria. España: Ediciones Díaz de Santos, S.A.; 1994.

10. Etcheverry G. Gestión de Calidad en Laboratorios Clínicos: Implementación de Gestión Clínica. Revista Bioanálisis. 2006; 11: 43-6.

11. Cabrera A, Bello L, Londoño J. Calidad Percibida por usuarios de Hospitales del Programa de Reestructuración de Redes de Servicios de Salud de Colombia. Rev. Salud Pública (Bogotá). 2008; 10(4):593-604.

12. Pérez-Tejada H. Estadística para las Ciencias Sociales, del Comportamiento y de la Salud. Tercera Edición. México: Cengage Learning Editores S.A; 2008. p. 729.

13. Amaro Sáenz BA [Internet]. Evaluación de la calidad de la atención al usuario externo del laboratorio clínico de los hospitales del IMSS en Aguascalientes. Universidad Autónoma de Aguascalientes Instituto Mexicano del Seguro Social. Disponible en http:// www.uaa.mx/investigacion/memoria/ponencias/mesa_cb/doc/amaro_saenz.doc Consultado 6 de enero 2009.

14. Seclen J, Darras C. Satisfacción de usuarios de los servicios de salud: Factores sociodemográficos y de accesibilidad asociados. Peru 2000". Universidad Nacional Mayor de San Marcos. Anales de la Facultad de Medicina. 2005; 66 (2): 127-141.

15. Siloaho M, Solveig L., Puhakainen E, Ulla-Riitta N. The effects of quality-management systems on external quality-assessment performance in Finnish clinical chemistry laboratories. Journal for Quality, Comparability and Reliability in Chemical Measurement. 2006; 11(5):238-245. 
16. Álvarez JP, Gualteros SM, Valderrama ML. (Internet) Factores que Influyen en la Calidad de la Atención al Cliente Externo que utiliza los Servicios Ambulatorios y Complementarios de la E.S.E Hospital San Rafael de Tunja. Colombia. Biblioteca Digital Las Casas. Fundación Index. 2006; 2 (2). Disponible en http://www.index-f.com/lascasas/documentos/ Ic0122.pdf Consultado 6 enero 2009.

17. Lemoine $C$, Lemoine $M$ [Internet]. Evaluación de la percepción del desempeño del Laboratorio en Salud Pública por parte de sus usuarios. Centro Nacional de Consultoría de Bogotá, Colombia. 2002. Disponible en: http://190.25.230.149:8080/dspace/bitstream/ 123456789/431/1/Informe.pdf Consultad0 20 de noviembre 2008.

18. Oja P, Kouri T, Pakarinen A. From customer satisfaction survey to corrective actions in laboratory services in a university hospital. Internacional Journal for Quality in Health Health Care. 2006; 18(6): 422-428.

19. Riveros J, Berne C. Análisis de la opinión de usuarios sobre calidad percibida y satisfacción con hospitales públicos: Estudio de casos desde la perspectiva del marketing. Revista Médica de Chile. 2007; 135:862-870. 